1995

\title{
Supralinear Photoconductivity of Copper Doped Semi-Insulating Gallium Arsenide
}

\author{
K.H. Schoenbach \\ Old Dominion University \\ R. P. Joshi \\ Old Dominion University \\ F. Peterkin \\ Old Dominion University \\ R. L. Druce
}

Follow this and additional works at: https://digitalcommons.odu.edu/bioelectrics_pubs

Part of the Electrical and Electronics Commons, and the Engineering Physics Commons

\section{Repository Citation}

Schoenbach, K. H.; Joshi, R. P.; Peterkin, F.; and Druce, R. L., "Supralinear Photoconductivity of Copper Doped Semi-Insulating Gallium Arsenide" (1995). Bioelectrics Publications. 247.

https://digitalcommons.odu.edu/bioelectrics_pubs/247

\section{Original Publication Citation}

Schoenbach, K. H., Joshi, R. P., Peterkin, F., Druce, R. L., S., M. M., H., S. K., .. T., K. S. (1995). Supralinear photoconductivity of copper doped semi-insulating gallium arsenide. Journal of Applied Physics, 77(10), 5208-5214. doi:10.1063/1.359269

This Article is brought to you for free and open access by the Frank Reidy Research Center for Bioelectrics at ODU Digital Commons. It has been accepted for inclusion in Bioelectrics Publications by an authorized administrator of ODU Digital Commons. For more information, please contact digitalcommons@odu.edu. 


\title{
Supralinear photoconductivity of copper doped semi-insulating gallium arsenide
}

\author{
K. H. Schoenbach, R. P. Joshi, ${ }^{\text {a) }}$ and F. Peterkin \\ Physical Electronics Research Institute, Old Dominion University, Norfolk, Virginia 23529 \\ R. L. Druce \\ Lawrence Livermore National Laboratory, Livermore, California 94550
}

(Received 17 November 1994; accepted for publication 30 January 1995)

\begin{abstract}
We report on the intensity dependent supralinear photoconductivity in GaAs:Si:Cu material. The results of our measurements show that the effective carrier lifetime can change over two orders of magnitude with variations in the intensity of the optical excitation. A threshold intensity level has been observed and can be related to the occupancy of the deep copper level. Numerical simulations have also been carried out to analyze the trapping dynamics. The intensity dependent lifetimes obtained from the simulations match the experimental data very well. Finally, based on the nonlinear intensity dependence of the effective lifetimes, a possible low-energy phototransistor application for the GaAs:Cu material system is presented. (1) 1995 American Institute of Physics.
\end{abstract}

\section{INTRODUCTION}

Photoconductive switching has gained increasing interest in the pulsed power community due to its jitterfree operation on timescales extending into the subpicosecond range. Other advantages include the compactness of semiconductor based switches, isolation between the electrical and optical signals; flexibility in the detection sensitivity, large gains, and high power handling capacity. Besides silicon ( $\mathrm{Si}$ ), semi-insulating gallium arsenide ( $\mathrm{GaAs}$ ) is the most commonly used material for high power switching. ${ }^{1}$ The switching process, the generation of charge carriers in the semiconductor (and possible quenching of the photoconductivity), is controlled by high power laser pulses. Typically, laser energies larger than the bandgap of the semiconductor have been used for the optical switching process. This ensures a higher gain and conductivity value since both electrons and holes are photogenerated, contributing to the transport process. The higher gain, however, comes at the expense of slower switch turn-off times and larger electrical noise. Since semi-insulating semiconductors usually contain relatively large concentrations of defects and compensated impurities, which form deep levels in the energy gap, subbandgap radiation can also be used to generate free electrons or holes. Switching of semi-insulating GaAs with its large concentration of EL2 defects which have an activation energy of about $0.83 \mathrm{eV}$ is therefore possible with $\mathrm{Nd}$ :YAG and $\mathrm{Nd}$ :glass lasers whose photon energy is around $1.0 \mathrm{eV}$.

Sub-bandgap excitation as a means of triggering semiconductor switches presents potential advantages. Besides using less energy to generate free electrons, the large absorption depths associated with sub-bandgap radiation allow the ionization of larger volumes within the switch material at more uniform levels. For instance, in LEC grown semiinsulating GaAs, we have measured an absorption length of $0.8 \mathrm{~cm}$ for Nd:YAG radiation. ${ }^{2}$ Also, the carrier decay time for this type of switching becomes dependent on the trapping of one type of charge carrier rather than electron-hole recom-

\footnotetext{
${ }^{a}$ Electronic mail: rpj@eceson.ee.odu.edu
}

bination. Consequently, both direct and indirect semiconductors can be used for the photoconductive switching. Furthermore, in direct semiconductors, it becomes possible to obtain larger carrier decay times by ionizing defect/impurity levels having small capture cross sections for the charge carriers. ${ }^{3}$ This effectively leads to enhanced gain and can become an important consideration for low level signal detection applications.

Instead of relying on the "natural" defects in semiinsulating GaAs when using sub-bandgap radiation for switching, we have deliberately introduced well defined deep-level impurities into GaAs. Beginning with $n$-type silicon doped $\mathrm{GaAs}$, we have diffused copper $(\mathrm{Cu})$ which forms deep acceptors, for full compensation of the material. This experimental method described in detail elsewhere, ${ }^{4}$ allows the fabrication of semi-insulating GaAs starting with various initial $\mathrm{Si}$ concentration levels ranging between $10^{16} \mathrm{~cm}^{-3}$ and $10^{17} \mathrm{~cm}^{-3}$. Copper forms two acceptor levels, $\mathrm{CuA}$ and $\mathrm{CuB}$, where the first one has a hole activation energy of 0.15 $\mathrm{eV}$, and the latter one a hole activation energy of $0.44 \mathrm{eV}^{5}$ From the observed experimental results reported previously, ${ }^{3}$ it was concluded that besides the expected copper related hole traps, recombination centers were also formed after the processing of GaAs:Si:Cu. For instance, based on a rate equation model which was used for numerical comparisons with experimental data in the nanosecond temporal range, ${ }^{6}$ the concentration of recombination centers was estimated to be about $10^{16} \mathrm{~cm}^{-3}$. Now as is well known, several nonlinear phenomena can result in such photoconductors having deep centers which consist of both recombination levels and sensitizing centers (e.g., $\mathrm{CuB}$ in our case). ${ }^{7}$ Amongst the nonlinear effects are the optical quenching of photoconductivity and supralinear photoconductivity. The first of these effects has been utilized in high power (MW) photoconductive opening switches. ${ }^{8.9}$ The second effect, which corresponds to an increase in charge carrier lifetime with increasing photon flux, is the topic of this study.

The study of the intensity dependent nonlinear conductivity is important for several reasons. First, it helps deter- 
mine the intensity requirements for a high gain photoconductor used as a high power closing switch. Second, it also becomes possible to explore the possibility of utilizing such two-center GaAs:Si:Cu material for subnanosecond detection and as candidates for low noise, sub-bandgap phototransistors. Finally, by comparing the temporal development and decay of the supralinear photoconductivity with numerical simulation results, it becomes possible to extract useful material parameters and trapping cross sections. In the following, we will begin by describing the experimental details for the intensity dependent measurements of our $\mathrm{GaAs}: \mathrm{Si}: \mathrm{Cu}$ photoconductors. The experimental results will subsequently be reported and analyzed based on a rate-equation model. The simulations will yield quantitative data on some of the material parameters. Finally, based on the nonlinear intensity dependence of the effective lifetimes, we will discuss a possible low-energy phototransistor application for the $\mathrm{GaAs}: \mathrm{Cu}$ material system.

\section{EXPERIMENTAL SETUP}

The switch sample which was used for the experiment was fabricated by depositing a 1- $\mu \mathrm{m}$-thick layer of copper on the polished side of a $0.75-\mathrm{mm}$-thick silicon doped square wafer $(5 \mathrm{~mm} \times 5 \mathrm{~mm})$ with a silicon concentration of $2 \times 10^{16}$ $\mathrm{cm}^{-3}$. The sample was annealed for $17 \mathrm{~h}$ at a temperature of $558^{\circ} \mathrm{C}$. The front surface was then lapped with $10 \mu \mathrm{m}$ lapping film, and polished using a noncontact laminar flow polisher in a $0.5 \%$ solution of bromine in methanol. Goldgermanium contacts, $1 \mu \mathrm{m}$ thick, were evaporated onto the front face to form coplanar electrodes, $1.5 \mathrm{~mm}$ apart. The contacts were annealed for $8 \mathrm{~min}$ at $420^{\circ} \mathrm{C}$. The dark resistance was found to be $55 \mathrm{k} \Omega$, corresponding to a resistivity of $1.3 \times 10^{4} \Omega \mathrm{cm}$. The mobility of the slightly $n$-type sample was measured as $2890 \mathrm{~cm}^{2} \times \mathrm{V}^{-1} \mathrm{~s}^{-1}$. This was lower by almost a factor of 2 compared to the silicon doped GaAs before copper compensation.

The sample was mounted into a parallel plate $50 \Omega$ transmission line structure (strip line) of $48^{\prime \prime}$ length, with equal distance to the $50 \Omega$ charging cable and the $50 \Omega$ cable which is leading to the terminated input of a 7250 digitizer. The overall bandwidth of the diagnostic system is limited by the interconnecting cable to about $2 \mathrm{GHz}$. A diagram of the laboratory setup and a detailed description of the diagnostic circuits can be found elsewhere. ${ }^{10}$ A Quantel YG501-30 $\mathrm{Nd}$ :YAG laser was used to activate the GaAS:Si:Cu photoconductor. The passive mode locked laser produces a $200 \mathrm{ps}$ pulse with total pulse energies up to $50 \mathrm{~mJ} /$ pulse at a wavelength of $1.06 \mu \mathrm{m}$. The laser energy at the switch position was varied between $50 \mu \mathrm{J} \mathrm{cm}^{-2}$ and $20 \mathrm{~mJ} \mathrm{~cm} \mathrm{~cm}^{-2}$. Measurements of the absorption coefficient dependent on the intensity were also performed by recording the radiation which was transmitted through the sample and comparing it with the optical energy which was measured behind a mask of equal dimensions as the active switch area. The sample was dc biased at 15 and $30 \mathrm{~V}$, corresponding to average fields of 100 and $200 \mathrm{~V} \mathrm{~cm}^{-1}$, respectively.

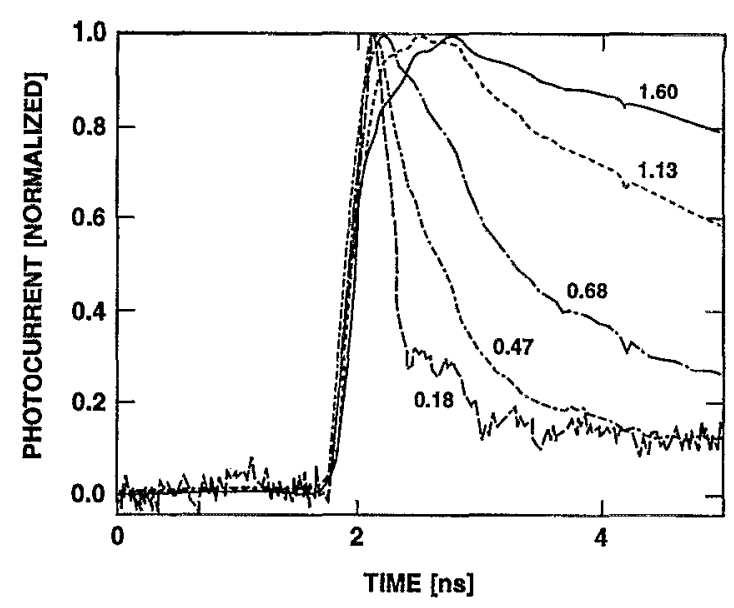

FIG. 1. Normalized temporal response of the photocurrent to laser excitation for laser energy densities ranging from $180 \mu \mathrm{J} / \mathrm{cm}^{2}$ to $1.6 \mathrm{~mJ} / \mathrm{cm}^{2}$.

\section{EXPERIMENTAL RESULTS}

The temporal response of the photocurrent (normalized) to the laser excitation is shown in Fig. 1 for laser energy densities, $E$, ranging from $180 \mu \mathrm{J} \mathrm{cm}^{-2}$ to $1.6 \mathrm{~mJ} \mathrm{~cm}^{-2}$. For low laser energy densities $\left(E<180 \mu \mathrm{J} \mathrm{cm}^{-2}\right)$, the fall of the photocurrent pulse follows the descending slope of the activating laser pulse, as shown in Fig. 2 for $E=51 \mu \mathrm{J} \mathrm{cm}{ }^{-2}$. The slower rise of the photocurrent compared to that of the activating laser pulse is attributed to the limited bandwidth of the current probe. At laser energy densities exceeding 200 $\mu \mathrm{J} \mathrm{cm}^{-2}$, the decay time, $\tau$, becomes dependent on the laser intensity. It increases approximately quadratically with rising laser intensity as shown in Fig. 3, and seems to reach a saturation value of $40 \mathrm{~ns}$ at laser energy densities of 5 $\mathrm{mJ} \mathrm{cm}{ }^{-2}$. These values of $\tau$ were obtained by measuring the slope of the current pulse right after the current peak. The data shown in Fig. 3 were obtained from two different voltages of 15 and $30 \mathrm{~V}$. Nearly identical decay times result from both data sets, because of the low values of the electric

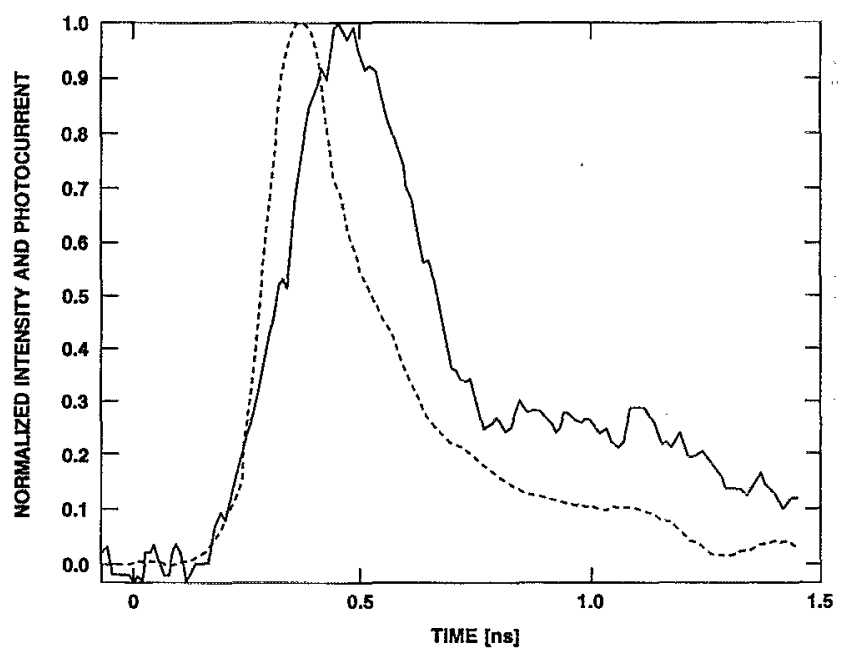

FIG. 2. The laser pulse shape and the temporal development of the photocurrent for an energy density of $51 \mu \mathrm{J} / \mathrm{cm}^{2}$. The $y$ axis is suitably normalized. 


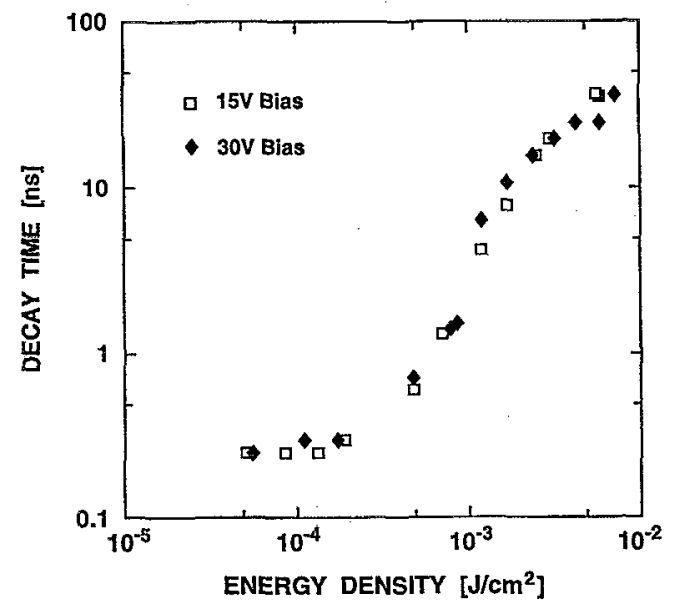

FIG. 3. Decay time as a function of the laser energy density. Data were obtained from the experimental time-dependent response of the photocurrents at two different bias voltages. At these low bias levels, the recombination processes are virtually independent of the electric field, leading to nearly identical decay times for the two voltages.

fields. The fields' intensities were 100 and $200 \mathrm{~V} / \mathrm{cm}$ for the two bias voltages. At these low levels, all of the recombination processes are independent of the electric field. Consequently, the decay times at a given laser energy density, were nearly identical within experimental error. A second type of current decay, with decay times in the microsecond range, becomes visible when the laser intensity exceeds $1 \mathrm{~mJ} \mathrm{~cm}^{-2}$. Figure 4 shows the long term current tail on a logarithmic scale for $E=3 \mathrm{~mJ} \mathrm{~cm}^{-2}$. The time constant of this exponential decay increases from $250 \mathrm{~ns}$ at $1 \mathrm{~mJ} \mathrm{~cm}^{-2}$ to about $1 \mu \mathrm{s}$ at $7 \mathrm{~mJ} \mathrm{~cm} \mathrm{~cm}^{-2}$.

The risetime of the photocurrent pulses $(10 \%-90 \%)$ is 200 ps for laser energy densities of less than $E=180$ $\mu \mathrm{J} \mathrm{cm}^{-2}$, and for any $E$ greater than $180 \mu \mathrm{J} \mathrm{cm}^{-2}, \tau$ is about $450 \mathrm{ps}$. The current amplitude, and consequently the photoconductivity, $\sigma$, at peak current stays constant in the intensity range from 50 to $180 \mu \mathrm{J} \mathrm{cm}^{-2}$ and then increases quadratically with the laser intensity as shown in Fig. 5, up to values

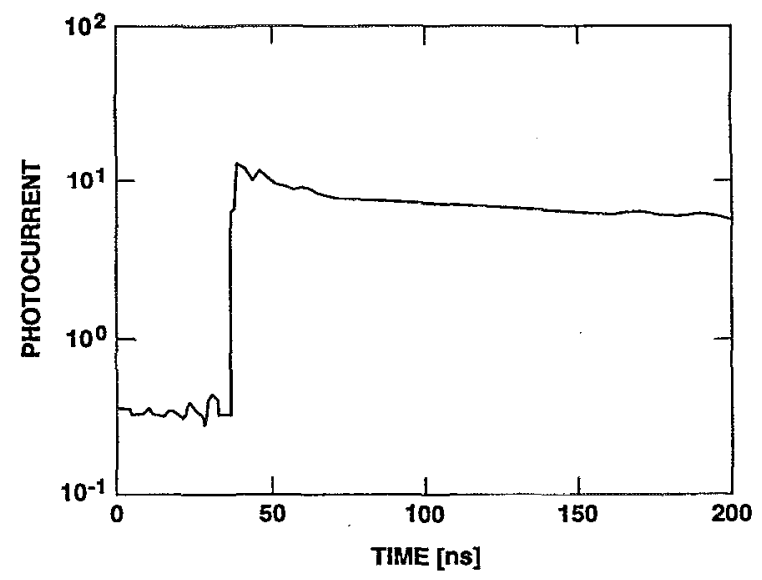

FIG. 4. Slow decay of the photocurrent observed experimentally for an energy density of $3 \mathrm{~mJ} / \mathrm{cm}^{2}$. The decay time corresponding to the given data is in the microsecond range.

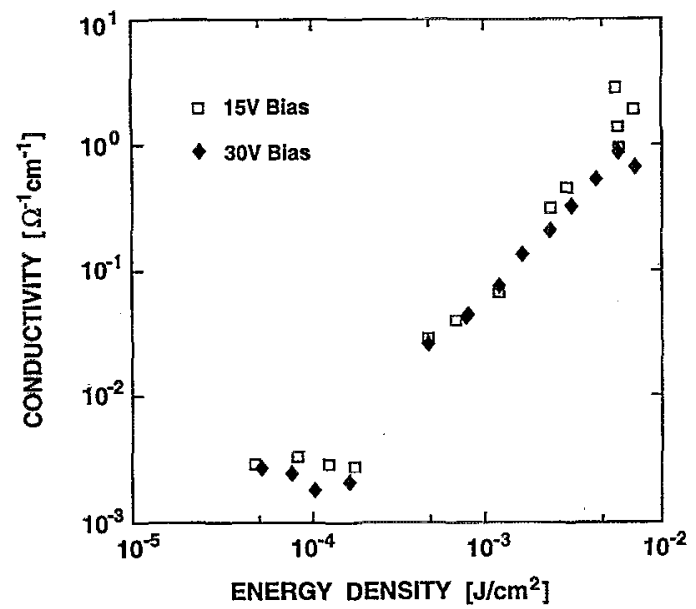

FIG. 5. Measured photoconductivity at peak current for various values of the laser energy density for two different bias voltages. The photoconductivity values are almost independent of the bias voltage as a result of the constant mobility at the low electric fields.

of several $\Omega^{-1} \mathrm{~cm}^{-1}$ at energy densities exceeding 5 $\mathrm{mJ} \mathrm{cm} \mathrm{cm}^{-2}$. The data shown in Fig. 5 were again taken at two biasing voltages of 15 and $30 \mathrm{~V}$. No discernable difference in the conductivity values was obtained for the two data sets. This is to be expected due to the low values of the electric fields, which were 100 and $200 \mathrm{~V} / \mathrm{cm}$, respectively for the two applied voltages. At these low fields, the electrical response is well within the ohmic regime leading to a constant mobility, and hence, nearly identical values of the measured conductivity for a given laser energy density. The absorption coefficient $\alpha$ was obtained from transmission measurements and was found to be $19 \mathrm{~cm}^{-1}$, corresponding to an absorption depth of $0.52 \mathrm{~mm}$. For the calculation of $\alpha$ it was assumed that absorption losses are large compared to the losses due to reflection such that quadratic terms in the reflection coefficient can be neglected in the equation for $\alpha .^{2}$ With an error margin of about $10 \%, \alpha$ was found to be independent of the laser intensity. The constant value of the photoconductivity at laser energies below $190 \mu \mathrm{J} \mathrm{cm}^{-2}$, therefore, cannot be attributed to an increasing absorption with reduced laser energy density.

\section{MODELING AND ANALYSIS OF THE PHOTOCONDUCTOR}

The observed increase in conductivity and current decay time for activation with laser energy densities above 180 $\mu \mathrm{J} \mathrm{cm}^{-2}$ can be understood by considering the shift in the Fermi level with increasing light activation. For a qualitative discussion, we concentrate on the two levels within the forbidden gap which are most important for the occurrence of supralinear photoconductivity: the recombination center, RC, and the $\mathrm{CuB}$ center. The $\mathrm{CuB}$ center is a hole trap with very small electron capture cross section. ${ }^{5}$ With increasing photoexcitation intensity the electron and hole quasi-Fermi levels shift towards the band edges. At low intensities, the hole Fermi level is above the $\mathrm{CuB}$ level. As a result, $\mathrm{CuB}$ acts as a hole trap and does not affect the recombination kinetics. With increasing intensity, the process of hole capture at the 
TABLE I. Parameters used for simulating the photoresponse in GaAs:Si:Cu.

\begin{tabular}{ll}
\hline \multicolumn{1}{c}{ Input parameter } & \multicolumn{1}{c}{ Value used (MKS units) } \\
\hline$N_{\mathrm{Cu}}$ (Copper density) & $1.85 \times 10^{22} \mathrm{~m}^{-3}$ \\
$N_{D}$ (Donor silicon density) & $2 \times 10^{22} \mathrm{~m}^{-3}$ \\
$N_{\mathrm{RC}}$ (Recombination center density) & $2 \times 10^{22} \mathrm{~m}^{-3}$ \\
$E_{\mathrm{Cu}}$ (Energy level of Copper) & $0.44 \mathrm{eV}$ \\
$E_{\mathrm{RC}}$ (Energy level of recombination center) & $0.71 \mathrm{eV}$ \\
$\sigma_{\mathrm{Cu}}^{n}$ (Electron capture cross section for $\mathrm{Cu}$ ) & $8 \times 10^{-25} \mathrm{~m}^{2}$ \\
$\sigma_{\mathrm{Cu}}^{p}$ (Hole capture cross section for Cu) & $2 \times 10^{-18} \mathrm{~m}^{2}$ \\
$\sigma_{\mathrm{RC}}^{n}$ (Electron capture cross section for $\mathrm{RC}$ ) & $2 \times 10^{-22} \mathrm{~m}^{2}$ \\
$\sigma_{\mathrm{RC}}^{p}$ (Hole capture cross section for $\mathrm{RC}$ ) & $2 \times 10^{-22} \mathrm{~m}^{2}$ \\
\hline \hline
\end{tabular}

$\mathrm{CuB}$ center is significantly weakened, and the center begins to assume the role of a recombination center. At very high laser intensities, the population of photogenerated holes can well exceed the density of available CuB centers. Under such a situation, $\mathrm{CuB}$ level will quickly become unoccupied due to the rapid hole capture immediately following the laser pulse. This depopulation will effectively terminate further hole removal at the $\mathrm{CuB}$ sites. The subsequent photoconductive decay will, therefore, be controlled largely by the midgap recombination centers.

In order to obtain quantitative information on the effect of varying photoexcitation on the photoconductivity of GaAs:Si:Cu, we have modeled the photoconductor using a rate equation code. ${ }^{6}$ The use of a zero-dimensional code is justified because of the low value of applied voltage in our experiments, which allows us to neglect space charge effects near the contacts and carrier drift current components. ${ }^{11}$ The concentrations and the capture cross sections for the various deep centers in GaAs:Si: $\mathrm{Cu}$, used in the code, are listed in Table I. Except for the recombination center, RC, the listed values were either obtained from our own measurements, or taken from the literature. ${ }^{6}$ The values for the recombination center were adjusted for a best fit with the experimental results. Modeling results for the temporal development of the photocurrent, with the photogeneration intensity as a parameter, are shown in Fig. 6. The curves were normalized to allow for a quick, qualitative comparison of the intensity dependent behavior. In keeping with experimental observations, the photocurrent is seen to have the slowest decay at the highest excitation intensity. Furthermore, the transient decay characteristics do not exhibit a simple exponential falloff. Instead, the photocurrent at all intensity levels is seen to decay much more rapidly during the initial stages. This trend again is similar to the experimental behavior. For instance, at the lowest intensity, the measured photocurrent reduces to about $30 \%$ of its initial value within the first $0.5 \mathrm{~ns}$, but subsequently decays at a much slower rate. Finally, the simulation results reveal residual conductivity which can also be seen in the measured data. For example, at lower laser intensities, the photocurrent is seen to approach $18 \%$ of its initial value. We believe the long lived conductivity is a result of electrons that cannot be quickly removed, and hence remain in the laser excited semiconductor. This can be seen more clearly by examining the temporal development of the free carrier populations and the trap occupancies.

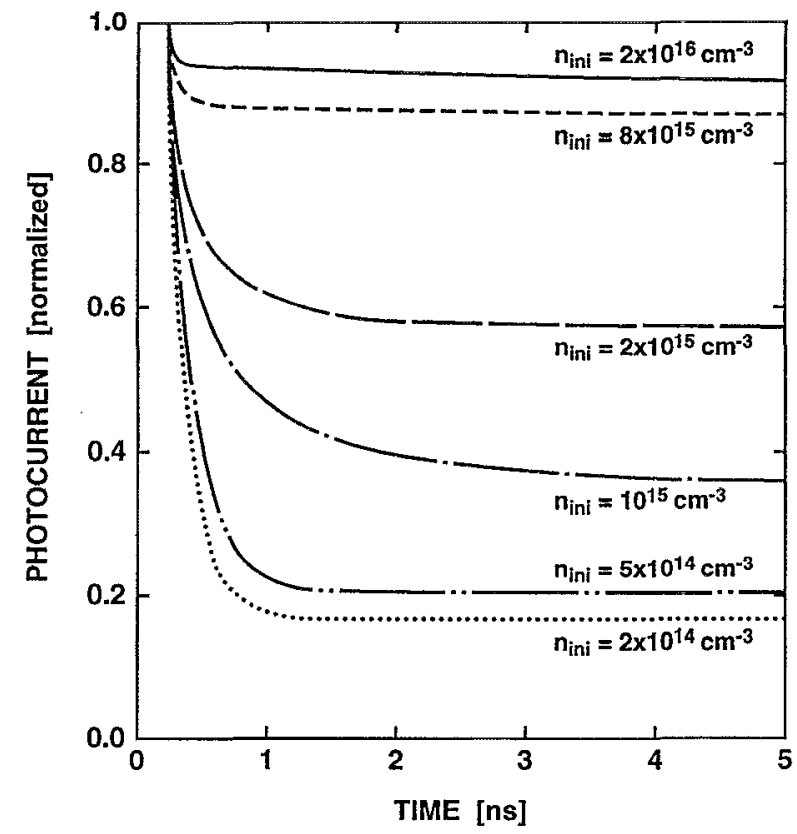

FIG. 6. Modeling results for the temporal development of the normalized photocurrent with the initial photogenerated carrier density as a parameter.

Figures 7(a) and 7(b) show the low- and high-intensity transients. At low laser intensity levels, the photogenerated free carrier densities are lower than the populations of either $\mathrm{CuB}^{-}$or $\mathrm{RC}^{-}$. The hole concentration immediately following the photoexcitation exceeds that of the electrons due to the larger density of states in the valence band. Also, the occupancy of the CuB level is greater than that of the recombination center $\mathrm{RC}^{-}$, because of its lower energy level. With time, the hole population begins to decrease rather quickly due to the large hole capture cross section of $\mathrm{CuB}^{-}$, and soon falls below the electronic density. As a result of hole capture, the occupancy of $\mathrm{CuB}^{-}$reduces marginally during this time, but this effect is only a minor perturbation from its initial state. On the whole, the available $\mathrm{CuB}^{-}$density is sufficient to provide a sustained hole removal mechanism at all times. The electrons, on the other hand, cannot be extracted quite as quickly since the electronic capture cross sections of $\mathrm{CuB}^{-}$ and $\mathrm{RC}^{-}$are much smaller. At the high intensity level, as shown in Fig. 7(b), the photogenerated electron and hole populations can significantly exceed both $\mathrm{CuB}^{-}$and $\mathrm{RC}^{-}$. During the initial phase, holes are captured and the occupancy of $\mathrm{CuB}^{-}$decreases rapidly. Owing to the large hole concentration, however, the fractional change in the its density is relatively negligible. With the substantially decreased occupancy, the $\mathrm{CuB}^{-}$can no longer provide an efficient channel for hole removal. As a result, the subsequent electron and hole densities remain quite high and are almost equal. The conductivity is then bipolar and the decay time is governed by the indirect recombination at the midgap RC level and direct electron-hole annihilation. Furthermore, it may be noted that though the above discussion pertains to a laser excited semiconductor, a similar decrease in the conductivity decay and possible persistent conductivity can be expected in semi-insulating GaAs at high biasing levels. 

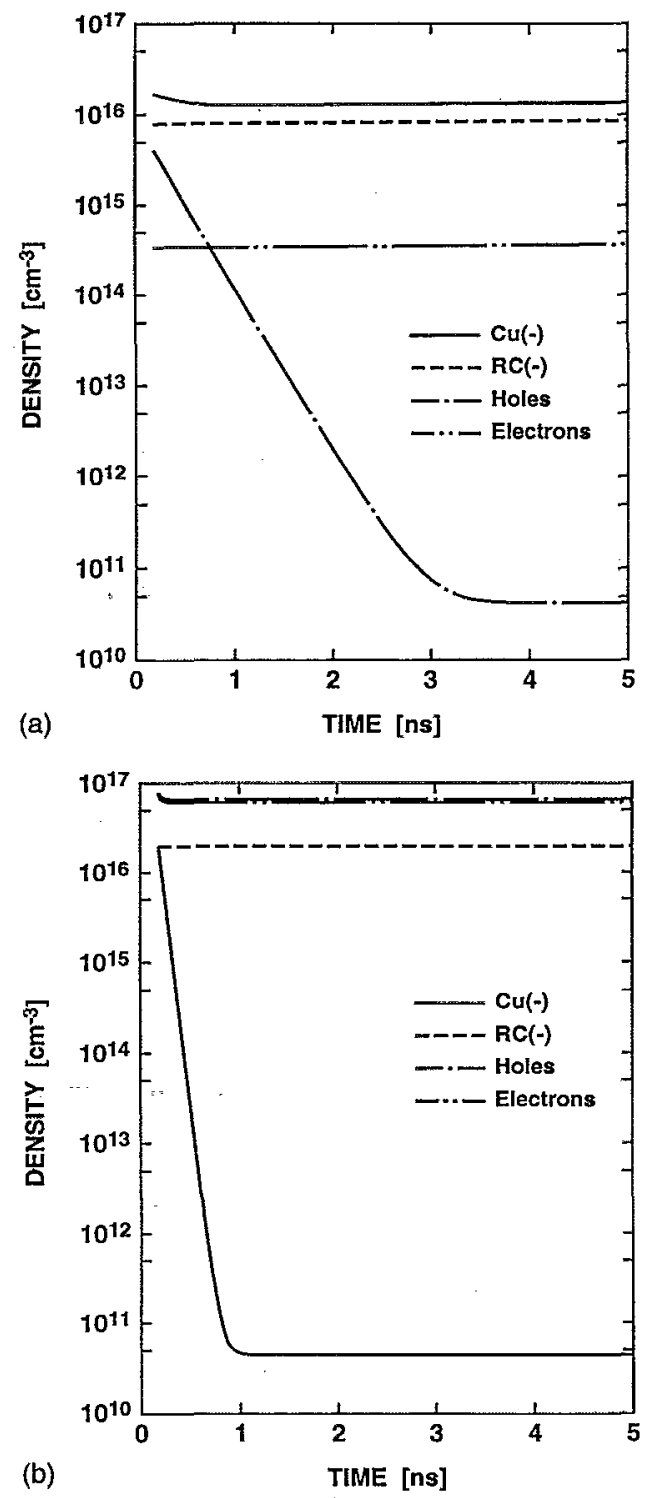

FIG. 7. Transient development of electrons, holes, copper, and recombination center populations obtained from the rate equation model. (a) Typical result for a low laser intensity situation, and (b) results for a high laser intensity. The electron and hole densities are almost indistinguishable in this case.

For a final comparison with the experimental data, photocurrent decay times were calculated from the simulation data for the various laser intensities. These values were evaluated from the initial decay transients. The time constants thus obtained, ranged from 0.445 to 48.7 . ns as the photon generation varied over two orders of magnitude. This result is exactly in the range of the observed experimental values, and the variation in photon intensity over this range is also by the same factor.. For a more direct comparison, both the theoretically calculated and the experimentally obtained time constants were plotted in Fig. 8. The $x$ axis was normalized to provide a more explicit comparison. As can be seen, both the theoretical and experimental curves match well over a span in which the laser flux changes by more than two orders of magnitude.

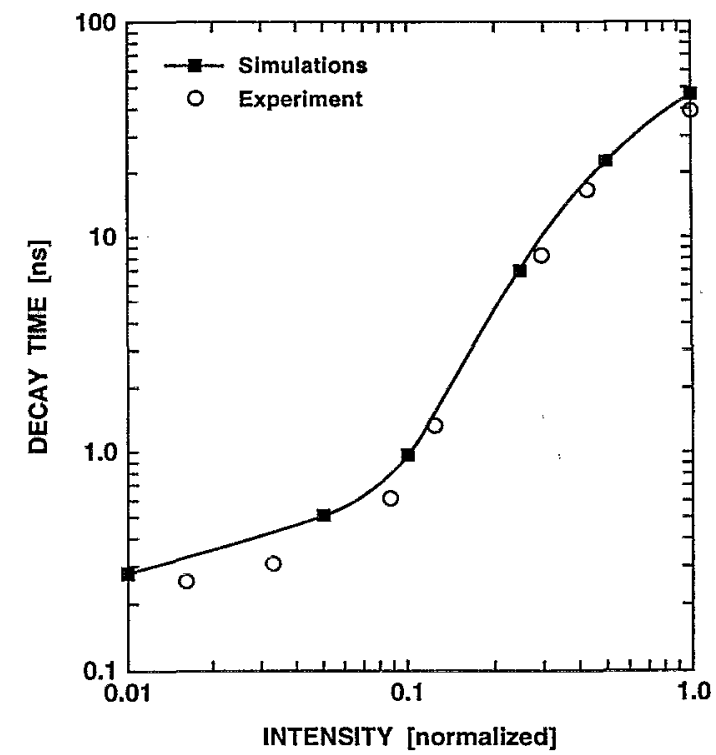

FIG. 8. Direct comparison between the calculated and experimentally obtained decay time constants. The $x$ axis is normalized. The experimental data presented here are the same as given in Fig. 3.

\section{APPLICATION}

The photoconductive response of the silicon-doped, $\mathrm{Cu}$ compensated GaAs material was shown to be intensity dependent. The effect is due to the shift in the hole quasi-Fermi level, which transforms the copper level from an effective hole trap to a recombination center. This property, in conjunction with the large energy $E_{\mathrm{Cu}}$ of the copper level, could be used to design low noise phototransistors for the detection and amplification of sub-bandgap radiation. This potential application along with its advantages are discussed in some detail below.

In principle, the $\mathrm{Cu}$-doped device would be similar to the usual $n-p-n$ structure with a floating base. ${ }^{12,13}$ The emitter and collector regions would consist of Si-doped $\mathrm{GaAs}$, and would be forward and reverse biased, respectively. However, the thin $p$-type base would consist of $\mathrm{Cu}$-doped $\mathrm{GaAs}$. By using copper, a deep level in GaAs, all of the acceptor states would normally remain unoccupied at room temperature. A simple calculation at $300 \mathrm{~K}$ easily confirms that $<0.3 \%$ of the deep $\mathrm{Cu}$ states would be filled. Hence, the entire base material, barring the depletion regions at either ends, would be available for strong absorption of sub-band gap radiation above the transition threshold $E_{\mathrm{Cu}}$. With such an arrangement, incident sub-bandgap radiation above $E_{\mathrm{Cu}}$ could be absorbed selectively only within the undepleted base with spatial uniformity. Consequently, the device would not suffer from any photoeffects within the depletion region. ${ }^{14}$ As in conventional phototransistors, transport of the photogenerated holes towards the emitter, would lead to electronic injection because of the increased forward voltage at the junction. This process would be responsible for the current gain. However, unlike conventional devices, using a $\mathrm{Cu}$-doped base region woúld provide some potential advantages as discussed next. 
First, the emitter efficiency and the base transport factors can both be expected to be higher than those for conventional phototransistors. The base transport factor for the $n-p-n$ structure would be large, for instance, because most of the mid-gap states would be occupied following the initial photoexcitation. This would effectively shut-off electronic capture. Though, theoretically, doubly charged defect states can be formed in the copper doped materials ${ }^{15}$ through electronic capture, the process is expected to be ineffective due to the repulsive Coulombic barrier to trapping. The low electron-capture cross-section of copper would also inhibit electronic trapping of the unoccupied states within the base. Next, the emitter efficiency would probably be improved for the structure, because the hole injection current into the emitter would not be quite as high. This would be a direct result of the high rate of hole capture within both the quasi-neutral and depletion regions of the $\mathrm{Cu}$ doped base. Furthermore, the device would also be well suited as a pulsed detector and promises favorable frequency response characteristics. A large frequency bandwidth is expected, for example, because of the inherent wave form sharpening of the hole pulse that is injected into the emitter. This sharpening would result since most of the holes in the leading edge of the hole swarm formed after photoexcitation would reach the emitter, while many holes at the trailing edge would either be trapped or would recombine with the incoming electrons. Since holes are heavier and have a lower mobility, sharpening the hole pulse should enhance the frequency characteristics and the pulsed response.

Finally, it must be mentioned that the intensity dependent trapping effect discussed previously can also be utilized in the functioning of the phototransistor structure. For weak pulses, it should be possible to attain a quick quenching of the photogenerated holes within the base given their short lifetime. Hence, the device should perform well as a fast, low-intensity pulse detector. The photocurrent pulses can be expected to be sharp and the device would exhibit a good frequency response. With increasing intensity, the photocurrent pulses would broaden. This effect, however, can be used for converting an amplitude modulated optical signal into a frequency modulated current wave form. Finally, for high intensity $\mathrm{cw}$ illumination, the phototransistor can be expected to provide a high gain due to the large carrier lifetimes.

\section{SUMMARY}

It had been shown previously ${ }^{4}$ that semi-insulating gallium arsenide, a photoconductor with ns response time, but consequently low photoconductive gain, can be converted into a high gain photoconductor, by doping it with silicon and compensating it with copper. The new material, GaAs:Si:Cu, has since been successfully used as a MW closing switch, and as a high power opening switch, utilizing the photoquenching effect. ${ }^{8}$ In this study, it was shown that the high gain effect is a threshold effect and is dependent on the photoactivation energy level. For the investigated material, laser energy densities on the order of $10 \mathrm{~mJ} \mathrm{~cm}^{-2}$ for a laser pulse duration of $200 \mathrm{ps}$ are required to switch into the high gain mode, where the current decay time can reach values of about $1 \mu \mathrm{s}$.

Lowering of the laser activating energy causes a nonlinear reduction in current decay time and in the value of the peak photoconductivity. For activating energy densities below $2 \mathrm{~mJ} \mathrm{~cm}^{-2}$, decay time, $\tau$, and conductivity, $\sigma$, vary quadratically with the laser energy. The lowest values of the current decay time are below that of the laser pulse decay time, which was on the order of 100 ps. The supralinear behavior of the peak photoconductivity and the charge carrier lifetime can be attributed to the shifts in quasi Fermi levels and the occupancy of copper states within the forbidden gap. Such behavior would always result if both recombination centers and traps were present with at least one dominant level having a large difference between the electron and hole capture rates. Obviously, the density of such a level would have to be large in order to create an observable effect. Modeling results, using a rate equation code, support this assumption and predict the intensity dependent lifetimes in keeping with experimental observations. The numerical simulations presented here, also yield quantitative values for the trapping cross sections and recombination center densities.

From the standpoint of applications, the GaAs:Si:Cu material is a promising candidate for high-power repetitive switching and photodetection. The gain of a photoconductor is defined as the ratio of the number of photocarriers crossing the contact area per unit time to the number of photons required to generate these carriers. Photoconductive gains of over a hundred have been obtained with contacts placed 1 $\mathrm{mm}$ apart and at applied fields below $5 \mathrm{kV} \mathrm{cm}^{-1}$. Another possible application for the GaAs: $\mathrm{Cu}$ material, is as a subnanosecond photodetector or phototransistor for low light intensities. As a phototransistor, one can expect the device to exhibit good frequency response with low noise at low intensities. Conversion from amplitude modulated signals to frequency modulated waveforms should also be possible. Finally, the relatively low electron effective mass for the material and the extended wavelength response up to $1060 \mathrm{~nm}$, might make this new material a competitor to the more conventional bulk Indium Phosphite and the quantum-well photodetectors. ${ }^{16}$

\section{ACKNOWLEDGMENT}

The research was supported in part by BMDO and managed by ONR. The program monitor is Gabriel Roy. This work was also performed in part under the auspices of the US Department of Energy by Lawrence Livermore National Laboratory under Contract W-7405-Eng-48. Computational support from Old Dominion University is acknowledged.

\footnotetext{
${ }^{1}$ M. D. Pocha and W. W. Hofer, Photoconductive Switching for High Power Microwave Generation, Proceedings of the Conference of Optically Activated Switching, edited by F. Zutavern (SPIE, Bellingham, WA, 1990), Vol. 1378, p. 2.

${ }^{2}$ R. A. Roush, M. S. Mazzola, K. H. Schoenbach, and V. K. Lakdawala, "Optical Quenching of Lock-On Currents in GaAs:Si:Cu Switches,"
} 
Records of the Nineteenth Power Modulator Symposium, San Diego, CA, June 1990, p. 339.

${ }^{3}$ M. S. Mazzola, K. H. Schoenbach, V. K. Lakdawala, and S. T. Ko, Appl. Phys. Lett. 55, 2102 (1989).

${ }^{4}$ M. S. Mazzola, K. H. Schoenbach, V. K. Lakdawala, R. Germer, G. M. Loubriel, and F. J. Zutavern, Appl. Phys. Lett. 54, 724 (1989).

${ }^{5}$ N. Kullendorf and L. Jansson, J. Appl. Phys. 54, 3203 (1983).

${ }^{6}$ S. T. Ko, V. K. Lakdawala, K. H. Schoenbach, and M. S. Mazzola, J. Appl. Phys. 67, 154 (1990).

${ }^{7}$ R. H. Bube, Photoelectronic Properties of Semiconductors (Cambridge University Press, Cambridge, 1992), Chap. V.

${ }^{8}$ D. C. Stoudt, J. S. Kenney, K. H. Schoenbach, R. A. Roush, S. Ludwig, and M. S. Mazzola, "Megawatt Operation of a Bistable Optically Controlled Semiconductor Switch," to appear in IEEE Trans. Electron. Devices (1994).

${ }^{9} \mathrm{~K}$. H. Schoenbach, "Optically Activated Opening of Copper Doped Gallium Arsenide Switches," in Optically Activated Solid State Switches, edited by F. Zutavern (Artech House, Boston, 1993), Chap. 6.
${ }^{10}$ M. D. Pocha and R. L. Druce, IEEE Trans. Electron Devices 37, 2486 (1990).

${ }^{11}$ R. P. Brinkmann, K. H. Schoenbach, M. S. Mazzola, R. A. Roush, and D. C. Stoudt, "Analysis of Time-Dependent Current Transport in an Optically Controlled Cu-Compensated GaAs Switch," Proceedings of the Conference on Optically Activated Switching II, Los Angeles, CA, January 1992, p. 262.

${ }^{12}$ J. C. Campbell, in Semiconductors and Semimetals, edited by R. K. WiIlardson and A. C. Beer (Academic, New York, 1985), Vol. 22, Part D, pp. 389-447.

${ }^{13}$ T. Moriizumi and K. Takahashi, IEEE Trans. Electron. Devices ED-19, 152 (1972).

${ }^{14}$ W. W. Gartner, Phys. Rev. 116, 84 (1959).

${ }^{15}$ For example, A. G. Milnes, in Deep Impurities in Semiconductors (Wiley, New York, 1973).

${ }^{16}$ For example, B. F. Levine, C. Bethea, G. Hasnain, V. O. Shen, E. Pelve, R. Abbott, and S. J. Hseih, Appl. Phys. Lett. 56, 851 (1990). 